\title{
Palaeomagnetic Evidence for Geomagnetic Field Reversals and Continental Drift
}

\author{
By S.K. RUNCORN \\ University of Durham, Newcastle upon Tyne
}

Inferences have been drawn from the study of the remanent magnetization of lava flows and red sediments as follows:-

(1) Over small stratigraphical thicknesses, a scatter of directions of magnetizatior is observed which in part is the result of a variation of the geomagnetic field about a mean, similar to the secular variation observed in historical times.

(2) Examining strata covering millions of years, reversals of polarity of magneti zation are observed, which have been interpreted either as self-reversals associatec with the magnetic properties of the iron oxide minerals or with reversals of the polarity of the geomagnetic field. If the latter, these reversals must occur at irregular intervals of time.

(3) Examining the whole of the geological column there is a slow shift of the axis of magnetization, which has been interpreted as polar wandering and continenta drift or that the geomagnetic field had non-dipolar components before the Tertiary Magnetostriction due to tectonic patterns has also been invoked to explain the data

Because the processes by which rocks become magnetized can never be reproducec with complete confidence in the laboratory, field tests must be devised to prove the truth of the geophysical hypotheses concerning the field reversals and relative dis placements of the continents and poles.

In case (1) we can hope to observe a variation of the amount of the geomagnetic secular variation with latitude.

In case (2), as was proposed by Runcorn (1956), the decisive test is to find a geological time in which reversals can be correlated over the world.

The reality of geomagnetic field reversals seems to have been established by the reversal of polarity at the Plio-Pleistocene boundary which has been observed in various parts of the world and by the absence of a reversal of polarity in Permian rocks: which in Australia, Europe and N. America, are magnetized in a direction consistent with a field of one polarity.

In case (3) the comparison of directions over a whole continent for one geological age establishes a consistency which rules out the hypothesis that the observed magnetizations do not reproduce the direction of the past geomagnetic field and that the differences in the polar wandering paths for the different continents can be better explained by the non-dipole hypothesis than by continental drift. Evidence from $\mathrm{N}$. American rocks will be presented relevant to these questions. 
The presence of a secondary magnetization along. the present dipole field in some sediments is an important limitation at present in these interpretations.

\section{DISCUSSION}

T. Nagata :

How would your explanation be for a continuous movement of the virtual pole of the earth's dipole from South to North or from North to South shown by Momose, Hales and Sigurgeirsson, independently, on basis of reliable rock-magnetism research?

S.K. Runcorn :

The work referred to is concerned with the mode of reversal of the geomagnetic field in Tertiary times. This reversal, which seems to take 10,000-100,000 years, might be accomplished by a decreasing to zero and then increasing into opposite direction or by the way Prof. Nagata suggests or by supposing the field generates strong non-axial harmonics in the period of reversal. But this sudden reversal in polarity reflects an unsteady electric current system in core, the 'non-dipole' hypothesis postulates the existence for some hundreds of million years of a field containing, on the average, higher harmonic terms.

A. Cox :

The physical argument of decoupling between core and mantle leads to the conclusion that the geomagnetic field should be axially symmetrical but not necessarily that of one particular multipole. Must we not test for the case of a general multipole consisting of terms of many orders of multipoles rather than for particular multipoles?

S.K. Runcorn :

I think it must always be reasonable to assume that the earth's main field is the sum of one or two of the lowest harmonics, for the core in which the field is generated is only just greater than half the mantle in radius and the higher harmonics fall off with radius more rapidly than the lower.

A. Cox :

The extensive and thorough studies by Angenheister of Miocene lava flows from Germany and by Aftab Khan of lava flows from the northern British Isles have poles that differ from the present geographic northern pole and are displaced toward the pole position of Roche, as shown on Prof. Thellier's slide. Perhaps our palaeomagnetic measurements are becoming sufficiently precise to enable us to detect a fine structure superimposed on the general smooth path of polar wandering.

K. Momose :

In the Enrei formation of upper Pliocene age, geomagnetic south pole determined from successive 20 lava flows wandered around the geographic south pole and the angular distances from the geographic pole were about $20-30^{\circ}$. 